\title{
O Que Faz um Artigo ser Mais Citado?
}

\author{
What Makes an Article be More Cited?
}

Conforme discuti em editorial anterior (Mendes-Da-Silva, 2020), a rejeição de artigos é algo com o qual devemos aprender, e inevitavelmente conviver, faz parte da rotina profissional do pesquisador. Mas isto precede um objetivo suposto comum a todos nós indivíduos pesquisadores da área de negócios: a publicação de nossas pesquisas, e que estas sejam julgadas relevantes para a sociedade. A esse respeito, uma das principais maneiras de aquilatarmos o nível de interesse nos nossos trabalhos é a citação que esses recebem (Garfield, 1955; Lee, Law, \& Ladkin, 2014), ou mesmo menções na mídia (Pulido, Redondo-Sama, Sordé-Marti, \& Flecha, 2018). Mas, afinal, o que faz um artigo ser mais citado (Hall \& Page, 2015)?

Na maioria dos casos, os cientistas dependem de literatura anterior relevante nos seus campos de pesquisa para o desenvolvimento de novas ideias. No entanto, não é sensato, tampouco viável, rastrear todas as publicações existentes, tendo em vista que o volume de trabalhos na literatura cresce extremamente rápido. Portanto, os pesquisadores geralmente seguem, ou citam, apenas uma pequena proporção de publicações pelas quais estão interessados (Bethard \& Jurafsky, 2010; Yan, Tang, Liu, Shan, \& Li, 2011).

\footnotetext{
'Fundação Getulio Vargas, Escola de Administração de Empresas de São Paulo, São Paulo, SP, Brasil.

${ }^{2}$ Editor-chefe da RAC - Revista de Administração Contemporânea.
}

Como citar: Mendes-da-Silva, W. (2020). What makes an article be more cited? Revista de Administração Contemporânea, 24(6), 507-513. https://doi.org/10.1590/1982-7849rac2020200167 
Isto é, a despeito da qualidade do trabalho de pesquisa realizado, um mesmo conteúdo informacional pode acabar por alcançar menor audiência essencialmente por questões de comunicação do resultado da pesquisa (Jacques \& Sebire, 2010). Assim, existem questões relativas ao artigo em si, e questões relativas ao periódico no qual se encontra publicado o trabalho de pesquisa. A esse respeito, por exemplo, Gargouri et al. (2010) defendem resultados empíricos em favor da relação causal positiva entre acesso aberto e número de citações, o que não é compartilhado por Kurtz e Henneken (2007). Um exame preliminar da literatura dedicada a estimar a quantidade de citações, condicional a atributos do artigo, permite listar, de maneira não exaustiva, variáveis como:

- Estar publicado na língua inglesa;

- Diversidade da audiência-alvo do tema abordado no paper;

- Idade do artigo - os mais antigos devem ser os mais citados;

- Índice H dos autores - quanto maior o índice H, mais citado tende a ser o paper;

- Estar em acesso aberto;

- Se utiliza dados, que estes sejam abertos, juntamente com o código utilizado;

- O título deve ser sugestivo do principal resultado da pesquisa;

- Os autores são oriundos de instituições sediadas em países diferentes;

- Fama - autor proeminente participando da autoria;

- Conexões do autor - autores socialmente mais conectados tendem a trazer mais citações;

- Autoridade - proeminência social no meio científico;

- Produtividade do autor;

- Uso de palavras provocadoras no título e no resumo, e.g.: inédito, revelador, robusto;

- O título do artigo deve ser amigável a máquinas de busca, contendo palavras-chave;

- O resumo deve trazer palavras-chave e reportar o principal resultado;

- Uso de palavras-chave ao longo do artigo;

- Trabalho no artigo após a sua publicação, compartilhando-o nas mídias sociais;

- O artigo atende a requisitos demandados para SEO (search engine optimization);

- Qualidade do processo editorial adotado pelo periódico onde foi publicado.
Comportamentos inadequados na autoria e/ ou na promoção de citações são uma realidade a ser apropriadamente endereçada, de modo a inibir epidemias de autoria honorária e citações artificiais (Jacso, 2006; Kovacs, 2013). A ocorrência da busca por citações, de maneira a negligenciar regras éticas, não é impossível de se constatar entre autores, editores e periódicos científicos. A RAC compartilha a visão de que a irrestrita observância a ditames éticos e respeitosos para a sociedade é algo que não está sob discussão.

\section{EVOLUÇÃO DO PROCESSO EDITORIAL DA RAC NO PERÍODO 2015-2019}

O processo editorial de um periódico deve ser tal que assegure a qualidade das publicações daí decorrentes. Não há dúvidas de que níveis mais elevados de padrão editorial tendem a demandar eficiência do processo em termos de uso de recursos, desde tempo até recursos financeiros. Um dos elementos particulares mais importantes para profissionalizar a gestão do processo editorial é a comunicação com o público interessado no periódico, que no caso da RAC temos buscado conduzir especialmente por meio dos editoriais componentes de cada edição. Conforme McGrail, Rickard e Jones (2006), um dos aspectos mais frequentemente considerados na caracterização de distinção de um periódico científico é a sua taxa típica de aceitação, ou de rejeição, de submissões recebidas.

Assim, em linha com práticas de gestão editorial contemporâneas, orientadas à transparência (Duflo, 2019), e de modo a permitir maior transparência acerca do processo editorial praticado nesta Revista de Administração Contemporânea (RAC), e ainda fornecer informações relevantes ao público interessado nesta revista (Hargens, 1988), neste editorial apresento uma visão resumida da evolução de indicadores do processo editorial da RAC (Mendes-Da-Silva, 2020) nos últimos cinco anos.

A Tabela 1 apresenta a evolução de números principais acerca do processo editorial da RAC. No painel A verifica-se que em 2015 foram recebidos quase 300 manuscritos entre artigos de pesquisa, casos para ensino e artigos tecnológicos. A quantidade de manuscritos recebidos pela RAC continuou crescendo desde então, chegando a 425 manuscritos em 2019 $(\Delta \%=42,14)$. Ao menos em parte, o crescimento da taxa de rejeição pode ser devido à decisão editorial pela redução do número de trabalhos publicados, que ocorreu gradativamente de quase 60, em 2015, para 40 , em 2019, tendo em vista o aumento da seletividade dos trabalhos publicados pelo periódico.

O painel B da Tabela 1 permite avaliar que, das 425 submissões recebidas em 2019 (ver painel A da Tabela 1), apenas 120 foram encaminhadas à peer 
review, o que sugere uma quantidade significativa de submissões que não passaram da fase de desk review. O motivo básico para a decisão de desk reject é a falta de clareza acerca da contribuição efetiva do manuscrito para o campo de conhecimento, assim como déficits de robustez característicos do procedimento metodológico adotado. Outros motivos menos expressivos estão detalhados no editorial da RAC anterior a este (Mendes-Da-Silva, 2020).

Tabela 1. Indicadores do processo editorial da Revista de Administração Contemporânea no período 2015-2019.

\begin{tabular}{ccc}
\hline Painel A: Relação entre manuscritos recebidos e publicados & & \\
\hline Ano & \# de documentos recebidos & \# de documentos publicados \\
\hline 2015 & 299 & 61 \\
2016 & 361 & 38 \\
2017 & 397 & 48 \\
2018 & 303 & 45 \\
2019 & 425 & 40 \\
\hline
\end{tabular}

Painel B: Tempo típico decorrido no processo editorial relativo aos trabalhos enviados ao peer review

\begin{tabular}{cccc}
\hline Ano & $\begin{array}{c}\text { \# de dias para decisão editorial } \\
\text { (média) }\end{array}$ & $\begin{array}{c}\text { \# de dias para decisão editorial } \\
\text { (mediana) }\end{array}$ & $\begin{array}{c}\text { \# de submissões enviadas } \\
\text { para peer review }\end{array}$ \\
\hline 2015 & 88 & 70 & 142 \\
2016 & 77 & 65 & 133 \\
2017 & 117 & 77 & 165 \\
2018 & 130 & 110 & 114 \\
2019 & 107 & 82 & 120 \\
\hline
\end{tabular}

Nota. Fonte: Escritório Editorial da Revista de Administração Contemporânea. Painel A: O número de documentos recebidos (submissões) para cada ano inclui todas as novas submissões entre $1^{\circ}$ de janeiro e 31 de dezembro de cada ano. Os documentos publicados para cada ano incluem todas as edições de cada volume, excetuando os Editoriais. Painel B: Os dados consideram o tempo de decisão para manuscritos com decisão. O número de submissões enviadas para peer review representa a quantidade de submissões que receberam ao menos um parecer avaliativo externo (revisão por pares de duplo-cego). A RAC mantém seu processo editorial apoiado na premissa da reciprocidade acadêmica (Mendes-Da-Silva, W. (2018a). Reconhecimento da contribuição do avaliador anônimo. Revista de Administração Contemporânea, 22(5), 1-13. https://doi.org/10.1590/1982-7849rac2018180281), e de maneira transparente reconhece publicamente as pessoas que colaboram no processo como revisores. Isto ocorre de duas formas complementares: a publicação anual de nominatas de indivíduos revisores, bem como a divulgação das identidades dos revisores de artigos publicados (mediante o consentimento dos respectivos revisores). ${ }^{1} \mathrm{Em}$ cada ano existe um conjunto de pessoas que atuam como revisores. Em 2015 foram 250 revisores, em 2016 foram 247, em 2017 foram 236, em 2018 foram 176, e em 2019 contamos com 213 indivíduos que compuseram o conjunto de voluntários que, em sistema double blind review, revisaram trabalhos submetidos. ${ }^{2}$ Inclui todos os tipos de documentos recebidos pela RAC, i.e., artigos de pesquisa, casos para ensino e artigos tecnológicos; não considera, portanto, Editoriais.

Ainda no painel B da Tabela 1 verifica-se que o ciclo editorial médio tem estado ao redor de 100 dias, sendo que aproximadamente $50 \%$ das submissões têm uma decisão final antes dos 90 dias. Ressalto que a RAC mantém seu processo de avaliação apoiado no princípio da reciprocidade acadêmica, conforme o qual solicitamos que os autores que submetem manuscritos aceitem avaliar trabalhos, em retribuição à avaliação minuciosa que seu trabalho deve receber por parte de outros colegas (Mendes-Da-Silva, 2018a). Nesse sentido, invariavelmente, há a necessidade de convidar ao menos cinco pessoas, até que tenhamos ao menos dois pareceres. Esse esforço editorial induz à inevitável elevação dos prazos típicos de avaliação no processo editorial. Esperamos que, à medida que haja maior disposição dos membros da comunidade para avaliar trabalhos, o ciclo de uma submissão seja sensivelmente menor.
Conforme a Tabela 2, as decisões de Revisão e Ressubmissão (R\&R) têm se tornado menos frequentes desde 2015, quando mais de $30 \%$ das submissões recebiam esse parecer, sendo aceito naquele ano pouco mais de $15 \%$ das 299 submissões recebidas. Assim, em 2018, das 303 submissões recebidas, pouco mais de $20 \%$ recebeu R\&R, sendo aceitas 14,5\%. Já em 2019 o quadro geral continua a mudar: recebemos 425 submissões, com 56 delas ainda pendentes; destas, 40,2\% receberam desk reject, contra $27,8 \%$ em 2015 . A situação líquida desse movimento é basicamente a seguinte: ao enrijecer os critérios de desk review estamos preservando um recurso escasso e valioso, e este é o revisor anônimo que realiza julgamentos cuidadosos e contributivos para os autores. Além disso, com uma resposta potencialmente mais célere aos indivíduos autores, promovemos a maior autonomia para que estes possam encontrar caminhos alternativos para publicar seus trabalhos. 
Tabela 2. Frequência de decisões editoriais atribuídas a manuscritos submetidos à Revista de Administração Contemporânea no período 2015-2019.

\begin{tabular}{|c|c|c|c|c|c|c|c|}
\hline $\begin{array}{c}\text { Ano de } \\
\text { submissão }\end{array}$ & $\begin{array}{c}\text { Aceitos } \\
\text { N (\%) }\end{array}$ & $\begin{array}{c}\text { Pendentes } \\
\text { N (\%) }\end{array}$ & $\begin{array}{c}\text { Rejeitados } \\
\text { N (\%) }\end{array}$ & $\begin{array}{c}\text { Desk reject } \\
\text { N (\%) }\end{array}$ & $\begin{array}{c}\text { R\&R } \\
\mathrm{N}(\%)\end{array}$ & $\begin{array}{c}\text { Arquivados } \\
\text { N (\%) }\end{array}$ & $\begin{array}{l}\text { Total }^{1} \\
\text { N (\%) }\end{array}$ \\
\hline \multirow[t]{2}{*}{2015} & 45 & - & 58 & 83 & 93 & 20 & 299 \\
\hline & $(15,1)$ & - & $(19,4)$ & $(27,8)$ & $(31,1)$ & $(6,7)$ & $(100,0)$ \\
\hline \multirow[t]{2}{*}{2016} & 36 & - & 57 & 155 & 89 & 24 & 361 \\
\hline & $(10,0)$ & - & $(15,8)$ & $(42,9)$ & $(24,7)$ & $(6,6)$ & $(100,0)$ \\
\hline \multirow[t]{2}{*}{2017} & 37 & - & 63 & 166 & 107 & 24 & 397 \\
\hline & $(9,3)$ & - & $(15,9)$ & $(41,8)$ & $(27,0)$ & $(6,0)$ & $(100,0)$ \\
\hline \multirow[t]{2}{*}{2018} & 44 & - & 52 & 132 & 61 & 14 & 303 \\
\hline & $(14,5)$ & - & $(17,2)$ & $(43,6)$ & $(20,1)$ & $(4,6)$ & $(100,0)$ \\
\hline \multirow[t]{2}{*}{2019} & 38 & 56 & 71 & 171 & 73 & 16 & 425 \\
\hline & $(8,9)$ & $(13,2)$ & $(16,7)$ & $(40,2)$ & $(17,2)$ & $(3,8)$ & $(100,0)$ \\
\hline
\end{tabular}

Nota. Fonte: Escritório Editorial da Revista de Administração Contemporânea. Entradas mostram o status em 20 de março de 2020 . Os números entre parênteses mostram as porcentagens relativas ao total de submissões em cada ano. Os manuscritos pendentes incluem submissões e revisões que estavam sob avaliação em 20 de março de 2020. Os manuscritos rejeitados representam as submissões rejeitadas após a revisão por pares de duplo-cego. Desk reject são manuscritos rejeitados sem revisão por pares, enquanto Revisar e Reenviar (R\&R) são manuscritos que foram devolvidos aos autores para correções naquele ano. ${ }^{1}$ Conforme relatado na segunda coluna da Tabela 1, no seu Painel A.

Tendo em vista o escopo atual da RAC, i.e., funcionar a partir de uma perspectiva regional e espírito interdisciplinar, buscando estar posicionada na vanguarda da inovação teórica e metodológica, dando boas-vindas a contribuições substantivas e empíricas, que investiguem e problematizem questões significativas de preocupação econômica, social e política, a revista recebe contribuições oriundas de pesquisas desenvolvidas em diversos campos de conhecimento na área de negócios. Entre as áreas de interesse especial da RAC encontram-se: gestão ambiental; produção, distribuição e consumo de recursos; política urbana; tecnologia, informação e conhecimento; mercados financeiros, de capitais, e finanças corporativas; cadeia de suprimentos; empreendedorismo; mercados de trabalho; e comportamento organizacional e individual. Essas áreas de pesquisa têm se apresentado à RAC, e vêm sendo publicadas nos últimos anos conforme ilustra a Figura 1.

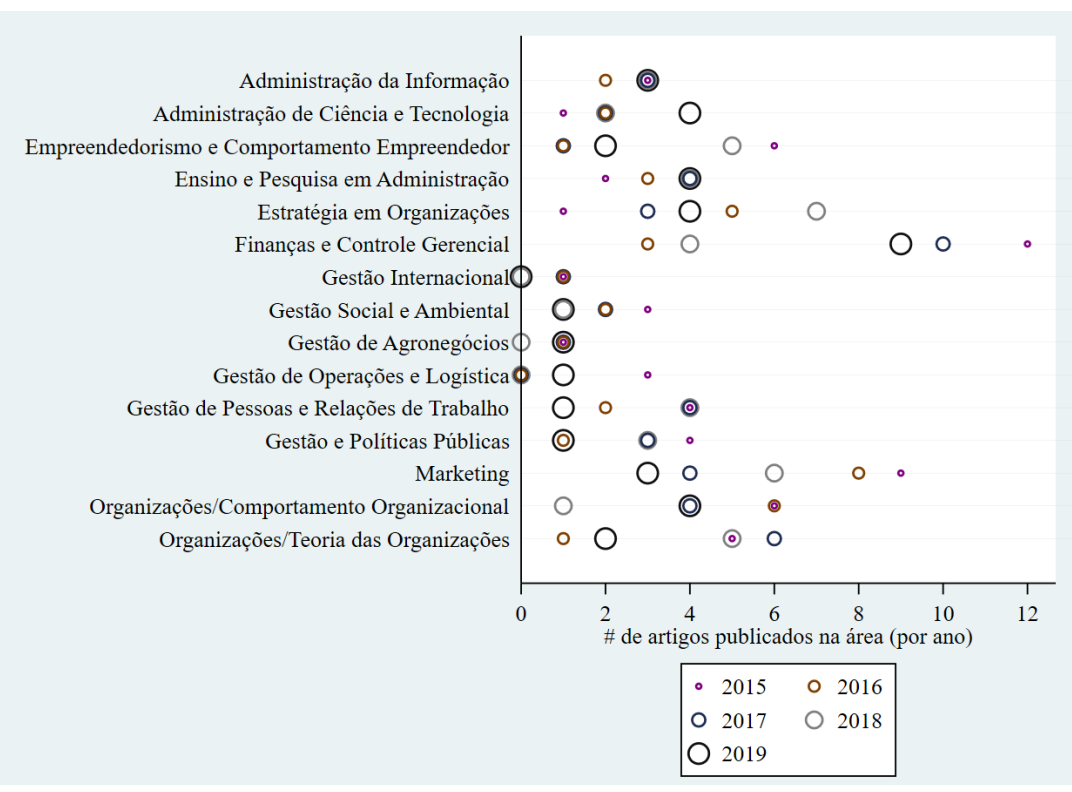

Figura 1. Frequência de artigos inéditos publicados pela Revista de Administração Contemporânea, por área de conhecimento em negócios (no período 2015-2019).

Fonte: Ilustração construída pelo autor, com base em dados gentilmente fornecidos pelo Escritório Editorial da Revista de Administração Contemporânea. Esta figura apresenta a quantidade de artigos publicados na RAC (por ano entre 2015 e 2019). 
Ao observar a Figura 1 verificamos que as áreas de gestão internacional, gestão de agronegócios e gestão de operações e logística compõem um subconjunto com menor presença na RAC nos últimos anos. Em paralelo, as áreas de estudos organizacionais, finanças e controle gerencial, e estratégia em organizações, juntamente com a área de marketing, aparecem entre as que mais têm contribuído para compor edições da RAC nos últimos cinco anos. Entre as áreas mais estáveis em termos de número de trabalhos publicados na RAC destaca-se administração da informação.

\section{INFORMAÇÕES ADICIONADAS AOS TRABALHOS PUBLICADOS PELA RAC}

Assim como os temas de pesquisa e os métodos empregados, o processo editorial evolui e sofre inovações. Nesse sentido, a RAC, nos últimos 24 meses, passou a publicar informações adicionais a respeito do processo relativo a cada trabalho publicado, mediante a adoção de um novo layout para os documentos publicados em um novo website. Assim, em cada artigo, desde então pode ser encontrado, em adição ao que já historicamente era reportado:

1. Divulgação do número de convites realizados a revisores anônimos, até que a decisão editorial em cada round de avaliação tenha sido tomada. Isto permite informar a comunidade acerca do esforço editorial envidado para que os trabalhos publicados sejam avaliados, e especialmente para que os autores possam aquilatar os motivos pelos quais, por vezes, o tempo decorrido na avaliação ultrapassa o esperado.

2. Adoção de resumo estruturado, de modo a facilitar a leitura do artigo em termos de informações tipicamente encontradas em um resumo de um documento científico.

3. Divulgação dos códigos de classificação conforme sistema originado pelo Journal of Economic Literature (JEL), de como permitir maior capilaridade e visibilidade dos trabalhos publicados pela RAC, no âmbito da comunidade de pesquisadores em negócios.

4. Divulgação da identidade dos editores e revisores do artigo, conforme sugere e discute Mendes-Da-Silva (2019a, 2019b). No caso dos revisores, sempre mediante seu consentimento prévio à publicação do artigo. Com esse procedimento a RAC espera contribuir para o reconhecimento público do trabalho voluntário desempenhado por colegas revisores. Além disso, permite a identificação de comunidades interessadas em determinados temas tratados nos artigos publicados.

5. Disponibilização de dados, materiais e códigos (Martins, 2020). Em outras palavras, a situação do artigo a respeito da política adotada de dados abertos, isto é: se o artigo possui dados abertos, onde estão os dados. No caso alternativo, qual a motivação dos autores para não compartilhar dados, materiais e códigos, juntamente com o artigo publicado.

6. Existência de financiamento da pesquisa publicada, de modo a reconhecer a contribuição de agências de fomento, ou qualquer outra fonte de recursos financeiros empregados na execução da pesquisa.

7. Método adotado para avaliação do artigo.

8. Método adotado para verificação de plágio.

9. Divulgação da contribuição suficiente para configurar a autoria dos artigos, de modo a reconhecer o esforço individual de cada autor, e ainda desestimulando principalmente a ocorrência de eventuais autorias cerimoniais, como destaca Rossoni (2018).

10. Existência de conflitos de interesse.

Essas iniciativas recentes da RAC, somadas a outras que virão, têm como principal motivação a promoção do impacto dos trabalhos publicados por este periódico, no sentido mais amplo dessa palavra 'impacto', não se restringindo ao número de citações na comunidade acadêmica. Nos últimos anos vimos o surgimento de métricas que intencionam mensurar esse tal impacto. Como proxy que vem ganhando espaço e reconhecimento, pode-se citar as mídias sociais (Mendes-Da-Silva, 2018b; Pulido et al., 2018).

Cabe não somente ao periódico o esforço por alcançar seu público, mas também aos demais players envolvidos no processo, e.g., autores que podem desenvolver suas habilidades de comunicação no nível individual, instituições de pesquisa que podem alocar recursos e construir estratégias e procedimentos dirigidos à comunicação ampla da pesquisa desenvolvida e publicada pelos seus colaboradores. Como nossos artigos têm sido recebidos pela sociedade? Essa é uma pergunta ao redor da qual todos nós devemos refletir e agir, sobretudo em momentos como o atual, quando as demandas da sociedade sobre a comunidade científica estão sensivelmente realçadas. 


\section{REFERENNCIAS}

Bethard, S., \& Jurafsky, D. (2010, October). Who should I cite? Learning literature search models from citation behavior. Proceedings of the ACM International Conference on Information and Knowledge Management, Toronto, Ontario, Canada, 19. https:// doi.org/10.1145/1871437.1871517

Duflo, E. (2019). Report of the editor: American Economic Review. AEA Papers and Proceedings, 109, 612-626. https://doi.org/10.1257/pandp.109.612

Garcia, D., Gattaz, C., \& Gattaz, N. (2019). The relevance of title, abstract and keywords for scientific paper writing. Revista de Administração Contemporânea, 23(3), 1-9. https://doi.org/10.1590/1982-7849rac2019190178

Garfield, E. (1955) Citation indexes for science: A new dimension in documentation through association of ideas. Science, 122(3159), 108-111. https://doi. org/10.1126/science.122.3159.108

Gargouri, Y., Hajjem, C., Larivière, V., Gingras, Y., Carr, L., Brody, T., \& Harnad, S. (2010). Self-selected or mandated, open access increases citation impact for higher quality research. PLoS ONE, 5(10), e13636. https://doi.org/10.1371/journal.pone.0013636

Hall, C. M., \& Page, S. J. (2015). Following the impact factor: Utilitarianism or academic compliance? Tourism Management, 51, 309-312. https://doi.org/10.1016/j. tourman.2015.05.013

Hargens, L. (1988). Scholarly consensus and journal rejection rates. American Sociological Review, 53(1), 139-151. https://doi.org//10.2307/2095739

Jacques, T. S., \& Sebire, N. J. (2010). The impact of article titles on citation hits: An analysis of general and specialist medical journals. Journal of The Royal Society of Medicine, 1(1), 1-5. https://doi.org/10.1258/ shorts.2009.100020

Jacso, P. (2006). Deflated, inflated and phantom citation counts. Online Information Review, 30(3), 297-309. https:// doi.org/10.1108/14684520610675816

Kovacs, J. (2013). Honorary authorship epidemic in scholarly publications? How the current use of citation-based evaluative metrics make (pseudo)honorary authors from honest contributors of every multi-author article. Journal of Medical Ethics, 39(8), 509-512. https://doi. org/10.1136/medethics-2012-100568

Kurtz, M. J., \& Henneken, E. A. (2007). Open Access does not increase citations for research articles from The Astrophysical Journal. ArXiv, 0709.0896. Retrieved from http://arxiv.org/abs/0709.0896

\section{Autoria}

\section{Wesley Mendes-Da-Silva*}

Rua Itapeva, 474, $8^{\circ}$ andar, 01332-000, São Paulo, SP, Brasil.

E-mail: rac.wesley.mendes@gmail.com

ㄴ https://orcid.org/0000-0002-5500-4872

* Autor Correspondente
Lee, H. A., Law, R., \& Ladkin, A. (2014). What makes an article citable? Current Issues in Tourism, 17(5), 455-462. https://doi.org/10.1080/13683500.2013.810611

McGrail, M. R., Rickard, C. M., \& Jones, R. (2006). Publish or perish: A systematic review of interventions to increase academic publication rates. Higher Education Research and Development, 25(1), 19-35. https://doi. org/10.1080/07294360500453053

Martins, H. C. (2020). Checklist evaluation for tutorialarticles: Clean code. Zenodo. http://doi.org/10.5281/ zenodo.3785747

Mendes-Da-Silva, W. (2018a). Reconhecimento da contribuição do avaliador anônimo. Revista de Administração Contemporânea, 22(5), 1-13. https://doi. org/10.1590/1982-7849rac2018180281

Mendes-Da-Silva, W. (2018b). Promoção de transparência e impacto da pesquisa em negócios. Revista de Administração Contemporânea, 22(4), 639-649. https://doi.org/10.1590/1982-7849rac2018180210

Mendes-Da-Silva, W. (2019a). Revisão pelos pares aberta e ciência aberta na comunidade de pesquisa em negócios. Revista de Administração Contemporânea, 23(4), 1-6. https://doi.org/10.1590/1982-7849rac2019190278

Mendes-Da-Silva, W. (2019b). Revisão pelos pares aberta e ciência aberta na comunidade de pesquisa em negócios, VIII reunião anual do programa SciELO Painel alinhamento dos periódicos SciELO Brasil com as boas práticas da ciência aberta. Zenodo. https:// doi.org/10.5281/zenodo.3572954

Mendes-Da-Silva, W. (2020). Lições que podem ser aprendidas da rejeição de um artigo. Revista de Administração Contemporânea, 24(4), 369-375. https://doi. org/10.1590/1982-7849rac2020200069

Pulido, C. M., Redondo-Sama, G., Sordé-Martí, T., \& Flecha, R. (2018). Social impact in social media: A new method to evaluate the social impact of research. PLOS ONE, 13(8), e0203117. https://doi.org/10.1371/journal. pone.0203117

Rossoni, L. (2018). Editorial: Produtivismo e coautoria cerimonial. Revista Eletrônica de Ciência Administrativa, 17(2), 1-8. https://doi.org/10.21529/RECADM.2018ed2

Uzzi, B., Mukherjee, S., Stringer, M., \& Jones, B. (2013). Atypical combinations and scientific impact. Science, 342(6157), 468-472. https://doi.org/10.1126/science.1240474

Yan, R., Tang, J., Liu, X., Shan, D., \& Li, X. (2011, October). Citation count prediction: Learning to estimate future citations for literature. Proceedings of the ACM International Conference on Information and Knowledge Management, Glasgow Scotland, UK, 20. https://doi.org/10.1145/2063576.2063757

\section{Conflito de Interesses}

O autor informou que não há conflito de interesses.

\section{Direitos Autorais}

A RAC detém os direitos autorais deste conteúdo. 


\section{CORPO EDITORIAL CIENTÍFICO E EQUIPE EDITORIAL PARA ESTA EDIÇÃO:}

\section{Conselho Editorial}

Anielson Barbosa da Silva (UFPB, João Pessoa, Brasil) Antonio Carlos Gastaud Maçada (UFRGS, Porto Alegre, Brasil) Ely Laureano Paiva (FGV, São Paulo, Brasil) Fabio Vizeu Ferreira (UP, Curitiba, Brasil) Maria José Tonelli (FGV, São Paulo, Brasil) Rogério Hermida Quintella (NPGA/UFBA, Salvador, Brasil) Valmir Emil Hoffmann (UnB, Brasília, Brasil) Wesley Mendes-da-Silva (EAESP/FGV, São Paulo, Brasil)

\section{Editor-chefe}

Wesley Mendes-da-Silva (EAESP/FGV, São Paulo, Brasil)

\section{Editores Associados}

Fabio Caldieraro (EBAPE/FGV, São Paulo, Brasil) Gilnei Luiz de Moura (UFSM, Santa Maria, Brasil) Henrique Castro Martins (IAG PUC-Rio, Rio de Janeiro, Brasil) Ismael Ali Ali (Kent State University, Ohio, EUA) Marcus Cunha Junior (University of Georgia, EUA)

Paula Castro Pires de Souza Chimenti (UFRJ/Coppead, Rio de Janeiro, Brasil)

Paulo César Matui (UniGranRio, Rio de Janeiro, Brasil)

Samy Dana (FGV/EAESP, São Paulo, Brasil)

\section{Corpo Editorial Científico}

André Luiz Maranhão de Souza-Leão (UFPE, Recife, Brasil) Aureliano Angel Bressan (CEPEAD/UFMG, Belo Horizonte, Brasil) Bryan Husted (York University, Canadá) Carlos M. Rodriguez (Delaware State University, EUA) Cristiana Cerqueira Leal (Universidade do Minho, Portugal) Diógenes de Souza Bido (Mackenzie, São Paulo, Brasil)
Erica Piros Kovacs (Kelley School of Business/Indiana University, EUA) Elin Merethe Oftedal (University of Tromsø, Noruega) Emilio Jose Monteiro Arruda Filho (Unama, Belém, Brasil) Fábio Frezatti (FEA/USP, São Paulo, Brasil) Felipe Monteiro (Wharton/University of Pennsylvania, EUA) Howard J. Rush (University of Brighton, Reino Unido) James Robert Moon Junior (Georgia Institute of Technology, EUA) John L. Campbell (University of Georgia, EUA)

José Antônio Puppim de Oliveira (United Nations University, Yokohama, Japão)

Julián Cárdenas (Freie Universität, Berlin, Alemanha)

Lucas Barros (FEA/USP, São Paulo, Brasil)

Luciano Rossoni (UniGranRio, Rio de Janeiro, Brasil)

M. Philippe Protin (Université Grenoble Alpes, França)

Paulo Estevão Cruvinel (Embrapa Instrumentação, São Carlos, Brasil)

Rodrigo Bandeira de Mello (Merrimack College, EUA)

Rodrigo Verdi (MIT Massachusetts Institute of Technology, Cambridge, EUA)

Valter Afonso Vieira, (UEM, Maringá, Brasil)

Wagner Kamakura (Jones Graduate School of Business, Rice University, Houston, EUA)

\section{Editoração}

Diagramação e normas da APA: Kler Godoy (ANPAD, Maringá, Brasil); Simone L. L. Rafael (ANPAD, Maringá, Brasil).

Periodicidade: Bimestral.

Circulação: Acesso totalmente gratuito.

Indexadores e Diretórios

Scielo | Redalyc | DOAJ | Latindex | SHERPA RoMEO | Cengage/GALE | Econpapers $\mid$ EBSCO $\mid$ MIAR | Proquest | SPELL | Cabell's |Ulrichs | CLASE | DIADORIM | ERIHPlus | EZB | EuroPub | OasisBR | WorldWideScience | Google Scholar | Capes/Qualis 